\title{
Fifty shades of Roboto: Text Design Choices and Categories in Multi-Scale Maps
}

\author{
Sébastien Biniek $^{\mathrm{ab},}$, Guillaume Touya ${ }^{\mathrm{a}}$, Gilles Rouffineau ${ }^{\mathrm{b}}$ \\ ${ }^{a}$ Univ. Gustav Eiffel, LASTIG GEOVIS, IGN, ENSG, F-94160 Saint Mande, France, sebastien.biniek@ign.fr, \\ guillaume.touya@ign.fr \\ ${ }^{b}$ ESAD GRENOBLE-VALENCE, gilles.rouffineau@esad-gv.fr \\ * Corresponding author
}

\begin{abstract}
The impetus induced by the development of multi-scale, multi-style maps calls for thinking our resources and protocols with greater interoperability. In the field of toponymy, this requires, in particular, thinking of categories and their structuring with more granularity. Assuming that typography, as a device for visualizing toponyms, is a tool whose potential is still under-exploited, we ask ourselves how the field of typographic design can improve our understanding of toponymic categories and help to structure them in a multi-scale logic. The approach adopted to answer this question is to analyse several existing maps to find good and bad practices. We identified different styles of place names and we built a typology of text features in the surveyed maps. The surveyed maps are the 1:35 000 scale, in IGN-France, OSM and GoogleMaps portals.
\end{abstract}

Keywords: typographic design, labels, cartographic knowledge, multi-scale maps

\section{Introduction}

Online mapping applications allow you to view maps at different scales and sometimes also offer different kinds of visualizations (e. g. mapping, orthophotos, orthoscans) The attendance statistics (Parsons, 2014) of these geoportals indicate that they are now widely consulted and are becoming the preferred cartographic navigation format. Nevertheless, it is important to note that these navigation tools, which are still quite recent, were quickly built from the cartographic representation conventions inherited from the classical reference designs, initially intended for paper use. While some of the actors design their multi-scale system globally, others still operate by aggregating existing maps (Dumont et al., 2016). Thus, depending on the scale consulted, map users may find themselves confronted with visualizations that differ greatly in terms of content and symbolization (Figure 1). This lack of coherence, which sometimes still remains across scales, can make navigation difficult and can negatively affect understanding of the symbologies used.These multi-scale navigation formats compel us to think in terms of scales, more in a continuum and call for us to think in terms of our resources and protocols with more interoperability.

In the field of toponymy, this requires, in particular, thinking of categories and their structuring with more granularity. The first superficial observations we have made, among the different map formats proposed in the geoportals accessible to navigation, the results obtained in our state of the art but also the prospects for improvements which are suggested, indicates that typography, as a device for displaying toponyms, is a tool whose parameters are still under-exploited. The virtual absence of standards for toponymic management in consortia such as the Open Geospatial Consortium also suggests that this deficiency would not only concern visualization issues but could be shared with issues related to the structuring of toponymic data.

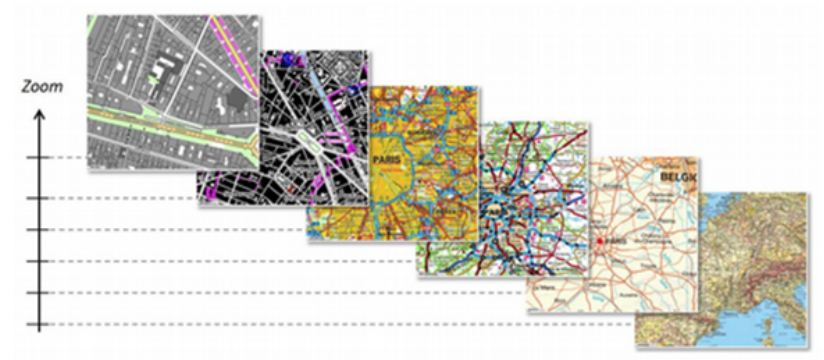

Figure 1. The different levels of a multi-scale map may present large differences - e.g. IGN France (Dumont et al., 2016).

Based on this observation, we ask ourselves how the field of typographic design can improve our understanding of toponymic categories and help to structure them in a multi-scale logic? The approach we are taking to answer this question is to analyze several existing maps for good and bad practices. Our approach model allows us to identify different styles of place names and involves the construction of a typology. We are testing it on a 1:35,000 scale corpus, IGN-France, OSM and Google maps.

In the first part, we highlight the importance of categories in understanding and structuring a map. In the second part, we will present some works connected to our issues. The third part is devoted to describing our survey methodology. Then, different aspect of our analysis are discussed in the third sections. Finally, the fifth part 
summarizes the results and discusses our perspectives.

\section{The importance of text categories in maps}

\subsection{Categorization}

Categorization is a fundamental cognitive process, which is involved in the perception and understanding of our environment. This allows us to distinguish a form, an object, a concept among others, and thus intervenes in our daily activity, allowing us to grasp our reality, understand it and anticipate it (Wilson and Keil, 1999). In order to build a knowledge of our environment, we organize space into different categories of objects and geographical structures (Debarbieux, 2004), by equivalence, differentiation or exclusion. Just as reading and writing rely on categorization skills for the perceptual processing of letters and words (e.g., recognizing the different available/existing spellings for the same letter), mapping rely on perception and categorization mechanisms, such as visual attention and visual recognition (Matlin, 2002). Visual recognition consists, during a visual perception experiment, in establishing a relationship between certain identified visual properties and similar elements previously memorized. In a cartographic context, it is a question of identifying geographical objects and structures based on the symbological choices that have been made.

\subsection{Learning cartographic knowledge}

Obviously, although the structuring of these visual languages is based on their ability to transmit information in an orderly way, our ability to understand them is not innate and requires to learn them. Shimron (1975) has shown that learning maps is facilitated by observing how the elements of a map are organized among themselves. When knowledge is mobilized to observe, categorization/discrimination comes into play and a belief/conviction about what is observed is formed. This is what Soltis (1966) called 'successful seing'. Gerber (1981) improves this concept by giving several examples to illustrate the different levels of complexity present in this phenomenon applied to the map:

- At the simplest level it consists of utilising one piece of knowledge, e.g. urban areas consist of many houses.

- At a more complex level it consists of the object seen as a multiple unit of related characteristics, e.g. an urban area consists of houses, roads and shops.

- At the most complex level, successful seeing consists of knowledge about an object beyond its identification, e.g. the urban area is seen as a dynamic system involving a range of inputs and a distinguishable range of outputs.

This allows him to develop a 3-level description model for the concept of successful seeing:

- (1). the perception-recipe level (pictorial);

- (2). the label level (pictorial-verbal) ;

- (3). the 'other knowledge about' level (verbal).

In this model, the label level is decisive, since it articulates the other two (verbal) \& (pictorial), and designates the labels as a key element that helps in learning and understanding the cartographic message, as also pointed out by Deeb et al. (2014). The study conducted by Ory et al. (2015), to identify the main graphical \& design characteristics perceived by map users to recognize an IGN-France topographic maps amongst other topographic maps, has stressed that labelling style is an important criterion. Consequently, typographic design plays a key role in the transmission and identification of the map message.

\subsection{Typographic design for labels/toponyms}

We are convinced that typographic design makes a significant contribution to making toponymic hierarchies visible and readable by visually structuring the similarities and disparity of geographical objects that are designated by toponyms. The typography applied to the cartographic context, as a reading experience and in these functional relationships, differs greatly from the so-called classical typography associated with continuous reading. Even if it is often considered that placement is an essential factor for readability in a map, and that there is a vast literature - devoted in particular to the development of algorithmic solutions to solve automatic placement of text (see for instance the review by Rylov and Reimer (2014)) - the field of readability research has paid close attention to the specificities of cartographic typography, when it has had to develop evaluation criteria to construct test protocols (Bartz, 1969).

The predominant factor in assessing the readability of a cartographic text design has long been associated with the notion of efficiency/effectiveness, i.e. the ease with which the content can be interpreted. As already proposed by Bartz (1969), who established the basic criteria for a typographic evaluation in cartography, it is necessary to identify a mapping task or activity that can be impacted by the variation of a typographic parameter. This activity must correspond to a real and common use of the map to determine if the typographical variation has an impact on the achievement of this activity. 'Type can be evaluated, then, not for itself but on the basis of how it affects the performance of that activity.' This impact must be measurable, evaluable, e.g. the speed, accuracy or ease with which the task is performed, in order to compare the effectiveness of different typographical arrangements/settings. Subsequent (e.g. Phillips, 1981) or more recent (e.g. Deeb et al, 2012) work on this subject incorporates these evaluation principles. In these types of protocols, the implementation often requires isolating the issues according to the parameter to be measured.

Evaluating typographical variables through efficiency tests seems particularly relevant to us, but we believe that to contribute to advancing the state of knowledge on these variables and their effectiveness, it may be relevant to study how they are used/exploited by card producers. This article explores the variations

The purpose of this paper is to examine how some map producers use typographical variables in their maps through their analysis and categorization. We assume that this type of information could allow a better definition of the categories of toponymic objects in order to facilitate and improve their typographic use. 


\section{Towards labels categories}

\subsection{Related Work}

For this research we consider approaches that have addressed the notions of categorization, and that in a way demonstrate an ontological will. The themes and issues that we propose to explore in this paper can be related to the existing approaches in the field of visualization and the definition of cartographic style, which aims to understand and qualify the relationships that exist between the production process and what visually distinguishes and characterizes one cartographic production from another. To this dialectical relationship of the problem - the visual characteristics resulting from cartographic operations - some research adds the factor of intention and highlights the need to provide a description and explanation of the objectives and means of production put in place to achieve them. Bucher et al (2007) propose, among other things, to explicitly represent these objectives according to the scale, the characteristics represented (represented features) but also to establish the relationships between these features and the reading modalities.

The study by Kent and Vujakovic (2009) is an important reference point for the field because their quantitative analysis deals with an extensive corpus of European topographic maps, produced by twenty national mapping Organizations (NMOs). Their selection criteria for the study focus on different points: number of symbols, colors of the themes, color of the "empty space", visual hierarchies and also make an analysis of the lettering. Their research highlights the existence of stylistic diversity. Their methodological bias is based on a spatial analysis that excludes the spatial characteristics of the represented space - the different NMOs do not have enough common mapped space that can potentially be analysed -, the processes used to construct the maps analysed and the perception of the symbology that users may have of them. Typography is analysed marginally, however, it is considered to be a category of symbol that can 'suggest a visual hierarchy'. Their inquiry uses a flexible and very generalist categorization for typography with a range (Figure 2) used for a typology of labels organized into five categories: settlements, relief features, rivers, and legend text.

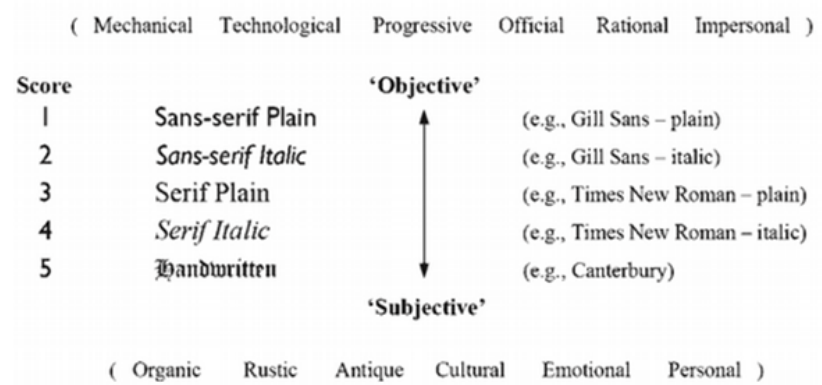

Figure 2. A continuum for classifying type styles on topographic maps (Kent and Vujakovic, 2009)

Their analysis verifies certain conventional typographical practices: black colour is used for all labels except hydrography; italics and cyan colour for hydrography ; increasing variation in text size and capital letters to suggest the importance of settlements and certain hydrographic areas ; roman sans-serifs for settlements labels and legend text ; two types of visualization commonly accepted for orography: one half of the countries use a sans serif font and the other a serif italic font. But more distinctive and marginal practices are also noted: left leaning fonts (also called: inverted italic, backslanted) for hydrographic symbology in Finland, Germany and Norway; serif italics on the entire toponymic range in Austria, Portugal and Switzerland. The indexing and scoring system implemented by Kent and Vujakovic also reveals a global trend: sans-serif characters tend to be used more uniformly on the map, rather than in a specific and punctual way.

Guidero (2013) also chose to focus on the salient graphic features that characterize a national topographic map style, but choosing typography in the maps as the central subject. His diagnosis points out that digital typography, with its status as a program in its own right, brings issues of compatibility and interoperability. It documents the fact that this type of problem motivated, among other things, the choice of typographies for the redesign, carried out between 2011 and 2013, of certain maps produced by the US Topo. 'Georgia and Trebuchet are legible, support extended character sets for diacritics, and are widely available on MacOS and Windows. Most users will have no trouble displaying labels set in these typefaces, as they will not have to install a typeface.' Nevertheless, Guidero reminds us that using system fonts has the disadvantage of using a visual signal that is already widely used in other contexts, and can therefore 'dilutes the identity of any design using it'. She also suggests that the use of open source licenses can be the solution to provide a custom font while eliminating all copyright restriction and embedding issues.

Brath and Banissi (2016) thought of typographical attribute issues as visual channels for coding differentiation and prioritization within an information system, focusing on different levels of efficiency, (e.g. bold or italic characters can be visually detected almost immediately, while differences between sans serif characters are difficult to perceive if the two characters used are very similar,). The distribution of these attributes by selected visual channels that Brath and Banissi has made is summarized in Figure 3. 'The diamonds indicate the primary visual channel that the font attribute uses, with a small diamond indicating a secondary visual channel that may be used. The pre-attentive potential column indicates whether the font attribute is likely to be perceived pre-attentively (i.e. perceived almost immediately, ahead of active focused attention) based on the properties of the underlying visual channel.' 




Figure 3. Font attributes relation to visual channels and encoding (Brath and Banissi, 2016).

\section{Description of the Survey}

\subsection{Choice of Criteria to Compare Maps}

The subjects of this study being, firstly (a) toponymic symbolization, and secondly (b) the understanding of the categorical structuring of entities by decoding this toponymic symbolization, optimizing the similarity factor between the different maps studied, seems to us to be the essential concern in our methodological approach, to articulate these two objectives. To achieve maximum consistency in our body of selected maps, we considered it crucial to compare maps representing the same localities and therefore the same geographical objects, as it is obvious that certain factors such as scale and the subject being mapped could have a significant influence on the degree of symbolization. We have therefore made several choices of criteria to build our methodological approach, allowing us to respect our two main subjects of study (a \& b). These following complementary criteria and methodological principles are listed in a logical order to better communicate how the analysis protocol is constructed and structured.

\subsection{Scale}

Kent and Vujakovic (2009), distinguish two types of map analysis in their research: a vertical one that concerns an analysis of symbolization under the scale and author variables, and a horizontal one, the one that Kent and Vujakovic choose to adopt, that compares maps on a single scale. To build a protocol model, simple enough to be able to implement it and increase its complexity later, a horizontal study model was chosen, which therefore focuses on a single scale.

We choose the $1: 35,000$ scale, which corresponds to zoom level 14, because it provides a balance between representation and abstraction. According to the classification identified by Lacoste (1980), which breaks down geographical representations into a range of scales including six orders of magnitude, the 1:35,000 scale is at the heart of the fifth group, which corresponds to maps using scales from 1:20,000 to $1: 50,000$. This fifth group corresponds to the scales of use of topographic maps and is adapted to their functions. To the extent that we focus on a single scale in this study, the $1: 35,000$ representations seem to us to reflect a balance, which may be relevant to question.

\subsection{Common map areas}

To have a relevant comparison, we believe that the corpus of maps should allow to evaluate visualizations relating to the same geographical areas. This type of criterion would, in particular, facilitate the observation of the second topic of study (b), which is concerned with the categorization of toponymic features. While choices in terms of structuration and organization of georeferenced data may vary from one mapping agency to another, comparing areas with potentially similar geographical objects minimize this effect, while at the same time providing an insight into the choices that are made in terms of content selection. However, in the situation where geoportals belong to national mapping agencies, the territory covered by the representation generally corresponds strictly to their respective national territory. There are therefore few or no shared geographical areas, the only exceptions being in some border areas.

The bias of this study is to use the "international" geoportals (e.g. GoogleMaps, OSM, Bing) as a reference and to observe the similarities and disparities of the NMOs through them.

\subsection{International Geoportals Compared to NMO's}

To carry out this study, we decided to rely on two international geoportals: Google \& Open Street Map. We believe that it is relevant to use them as references because of their significant global influence but also because their cartographic practices result from fundamentally different economic, political, organizational models.

\subsubsection{Map (1), Google}

Google, in terms of typographic management, may reflect a more corporatist approach, although the typography that is used for its cartographic visualization (Roboto) is widely distributed, under a free and open-source license.

\subsubsection{Map (2), OpenStreetMap}

OpenStreetMap, is a more transparent approach to data structuring but reflects a more community-based, empirical organization, which can be entropic. The desire to prioritize content seems much less strong than the tendency towards qualification, diversification and even densification in some places, which structures the exchanges of the OSM contributor community.

\subsection{Similar geographic objects}

Rather than using border areas to compare geoportals with each other, we use international geoportals as a comparator, which implies that cartographic representations studied from one country to another contain similar geographical objects. It is imperative to ensure a close correlation between the choice of geographical objects and the observation scale. The geographical objects that we consider most appropriate to observe through a 1:35000 representation, and more 
broadly with the scales belonging to the 5th group according to Lacoste's classification (1980), are the large urban areas.

The type of urban settlements we choose are cities that for the most part have a total population of between 100,000 inhabitants, an area ranging from 40 to $80 \mathrm{~km} 2$, and a population density of around 2000/3000 inhabitants per $\mathrm{km} 2$.

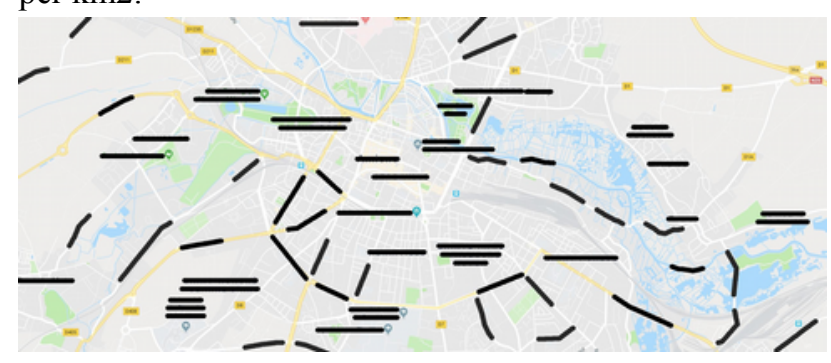

Figure 4. An example of the methodology used to count the toponyms present in the maps collected.

We are aware that from an urban, geographical or historical point of view, the comparison of the different urban entities chosen in this study may be clumsy. However, the construction of this method, although empirical, gives us great consistency. We also believe that our survey model should be able to cover a greater geographical diversity and extending this study to rural areas would be an interesting complement.

4.6 A common screen observation area

Using a common screen area is a necessary feature since the space dedicated to viewing the map representation varies from one geo-portal to another, depending on the navigation options offered by the sites that host the geoportals. We think it is wise to use a pixel space/window within which there is a systematic cartographic representation free of tools and information to assist cartographic navigation such as scale, zoom tools, etc. This area is defined from the navigation space of several geoportals that include both international geoportals and a wide range of NMOs, in order to be able to later implement our corpus. The common and fixed screen area also makes it easier for us to compare the font sizes used, based on the pixel unit. The methodology developed to exploit these screenshots and compare them consists simply in counting the toponyms by highlighting (Figure 4) the cartographic representations, attempting a distribution by geographical object categories.

\section{Analysis}

\subsection{Receiving/using data}

The data used to build the Google Maps and OSM repository for our study is derived directly from the methodology detailed in the previous section. In order to be comparable with maps from several NMOs, the corpus of maps for Google and OSM is based on a selection of one or two agglomerations for each respective territory of the NMOs we had planned to analyze. Despite the fact that the maps of these NMOs are not included in the study presented in this paper, this has allowed us to build a more solid corpus to understand the typographical hierarchies and toponymic categories for these two international geoportals and tolerates the continuation of this study, by implementing other NMOs.

\subsection{Categories}

To design a coherent typology (Figure 5) the classification had to evolve over time. The construction of broad categories (A: Inhabited Locality, B: Uninhabited Locality, C: Road and Transport Network, D: Hydro, E: Relief/Orography) was a necessary step to ensure the compatibility of the results between the three different maps of the panel. We deduced the text specifications (e.g. font, font size, case...) using different methods, depending on the geoportal. For Google, the method is purely visual; for OSM both stylesheets and a visual assessment were used; for IGN-France it is primarily visual, but supplemented by documentation on paper map specifications.

5.3 Use and distribution of typographic variables according to geoportals

\subsubsection{Google}

The character used by GoogleMaps is the Roboto, which was developed specifically by Google. It was implemented in 2013 on GoogleMaps and is also the default font of other Google services.

The textual specifications deduced for the category 'Inhabited locality' use several types of variables for the range of toponym instances that compose it. The size variable is used with great amplitude, but it is also supported by a differentiated use of font versions, letter cases, and colors. A large part of the instances are composed in upper case, with the exception of those at the extremes of the spectrum, i.e. the most and least populated. This type of marginal use is perhaps there to create a clearer distinction between median instances and toponyms belonging to other categories but having the 'cap height'. However, it is difficult to know what visualization functions are assigned to grayscales, since there is no gradual use of value.

With regard to the category of uninhabited locality, only the colorimetric variable is used and corresponds to a thematic qualitative use, although some toponyms are composed in greater proportions to indicate their importance.

\subsubsection{OSM}

The character used by OSM is Noto Sans, published by Google since 2012 under the Apache 2.0 license. They cover a very large part of Unicode characters, which certainly motivated its use. The major observation that can be made about OSM's typographical choices is that the size variable is practically not exploited. The difference between the maximum values for letter height is small: 5 pixels. This has the effect of homogenizing the overall impression left by the text. The size used is rather small but remains quite readable despite the resolution. The major disadvantage of this kind of choice also lies in the fact that it minimizes by correlation effect the impact of the use of other variables, insofar as it reduces the visibility of the signs that are the vectors of these other variables. 

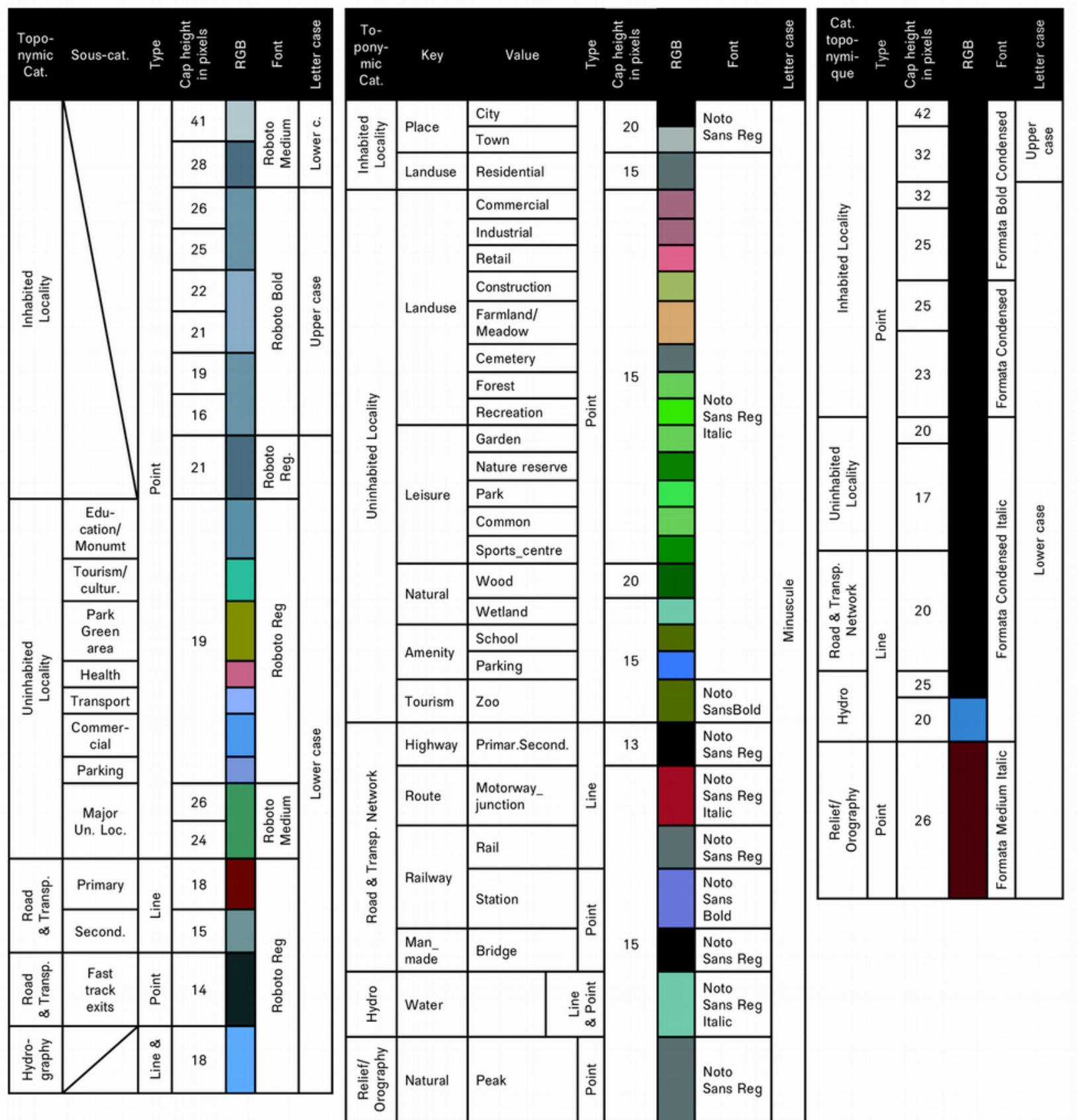

Figure 5. Our typology of text specifications deduced from IGN-France, OSM and GoogleMaps portals, at the 14th zoom level.

The most salient variable used is the shape differentiation, rendered by the use of italic. Italic is rather widely used to indicate uninhabited locality. This variable is systematically applied to the key attributes: leisure and landuse.

There is a very important granularity factor within the category of inhabited locality, whose rendering is ensured by the colorimetric variable of toponyms. This granularity reflects the granularity that exists in the structuring of data, for the same key there can be a great variety of value (e.g. within the key: landuse).

The granularity that exists within the uninhabited locality category is rather low compared to IGN-France or Google. It is perhaps once again related to the number of subcategories for inhabited locality in OSM features description. While it is usually quantitative stand data, which are associated with this type of category and correlated with the visual size variable.

\subsubsection{IGN}

Like the other geoportals observed, IGN uses a sans-serif character for its standard cartographic visualization : Formata, which is distributed under proprietary license. Nevertheless, what distinguishes it from others, is the use of a condensed version. The use of a condensed character in cartography is not marginal, it is a characteristic that is considered virtuous for the domain because it saves space The IGN map uses the size variable with a large amplitude by correlating it with a capital use for the most important localities to report quantitative differences related to population. It is a convention, but it is used in a sufficiently meaningful and prominent way to clearly perceive disparities. The difference between the maximum values for letter height is significant: 25 pixels. The use of the colorimetric variable is used to produce contrast and distinction, only two categories are affected 
by its use, hydrographic and orographic toponyms. For the most part, the typographical choices made by IGN are more straightforward and assertive than those of its international counterparts.

5.4 Occurrence on a common territory

Another aspect of the methodology used in this analysis is the ability to compare the choices made regarding toponymic symbolization for the same territory. We illustrate this aspect by an example applied to two agglomerations of equivalent size, located in France to use the IGN map in the comparison.

\begin{tabular}{|c|c|c|c|c|c|c|c|}
\hline \multicolumn{2}{|c|}{$\begin{array}{c}\text { Toponymic } \\
\text { cat. }\end{array}$} & $\begin{array}{c}\text { Inhabited } \\
\text { Locality }\end{array}$ & $\begin{array}{c}\text { Uninhabited } \\
\text { Locality }\end{array}$ & $\begin{array}{c}\text { Road \& } \\
\text { Transp. }\end{array}$ & $\begin{array}{c}\text { Hydro- } \\
\text { graphy }\end{array}$ & $\begin{array}{c}\text { Relief/ } \\
\text { Orography }\end{array}$ & Total \\
\hline \multirow{3}{*}{ Google } & Amiens & 15 & 9 & 31 & 2 & & 57 \\
\cline { 2 - 9 } & Limoges & 12 & 7 & 34 & 3 & & 56 \\
\cline { 2 - 9 } & Amiens & 4 & 6 & 21 & 6 & & 37 \\
\cline { 2 - 9 } OSM & Limoges & 20 & 1 & 29 & 3 & & 53 \\
\cline { 2 - 9 } & Amiens & 18 & 19 & 12 & & 1 & 50 \\
\hline \multirow{3}{*}{ IGN } & Limoges & 60 & 12 & 1 & 3 & & 75 \\
\hline
\end{tabular}

Figure 6. Distribution of toponymic categories according to the geoportals studied for the agglomerations of Amiens and Limoges, at the zoom level 14.

Unlike the specification table (Figure 5) which is built on data from about ten screenshots from different regions (for Google \& OSM), this sample presents a much more local data set. Even if there is too little data to draw clear lessons from it. It does, however, make it possible to distinguish certain trends that are emerging.

For Google, we can see a consistency in the distribution of the number of toponyms according to categories in each of the agglomerations. If there are no major differences in the total number of toponyms, depending on the area mapped or the geoportals, divergent trends can be distinguished according to the categories for OSM and IGN-France. Regardless of the area, OSM shows few toponyms belonging to the uninhabited locality category but provides many more toponyms related to the road and transport network. For its part, IGN-France shows a significant gap between road and transport network and the inhabited localities.

\section{Conclusion}

This kind of study provides a reasonable understanding of how the survey model implemented can provide relevant results for understanding practices related to text visualization. It can be apply to different scales. The choices in terms of typographic visualizations can help to guess the producer's intentions, and are linked to the identity they are supposed to reflect (e. g. the global choices made by OSM in their standard visualization are more reminiscent of the intention to reveal the existing resources and granularity in the OSM database). This study only partially shows the existing relationships, in terms of toponymic visualization and toponymic structuring, between different online map geoportals. We believe that our essential contribution lies in the construction of a relevant proposed analytical model. For the future, developments should be used with a larger corpus of maps and should articulate its horizontal architecture with a vertical approach, in order to assess the coherence and distribution of toponymic categories and their visualizations in multi-scale navigation. Then, we want to use the knowledge extracted from this study to design multi-scale, multi-style typographic design to improve the navigation fluidity between scales and styles. Increasing the level of complexity of the cartographic environment requires us to think more about the visualization and symbology of toponyms from a systematic perspective and therefore to identify in a more precise and clear way the toponymic categorization, both at the level of the objects themselves and the attributes that characterize them, in order to initiate an ontological approach as Mathian et al. (2014) suggests, and to place ourselves at the interfaces of typography, geomatics and informatics. An ontology derived from our typology could be used to control consistency of our text categorization across scales, which should improve the fluidity of navigation in these maps.

\section{References}

Bartz, B. (1969). Search: an approach to cartographic type legibility. Journal of Typographic Research. 3. pp. 387-398.

Brath, R., and Banissi, E. (2016). Font attributes enrich knowledge maps and information retrieval: Skim formatting, proportional encoding, text stem and leaf plots, and multi-attribute labels. International Journal on Digital Libraries, 18. pp. 5-24.

Bucher, B., Jolivet, L., Buard, E. and Ruas, A. (2007). The need for Web legend services., 7th International Symposium on Web and Wireless GIS (W2GIS), Lecture Notes in Computer Science, Springer ed. by Ware, J. M. and Taylor, G. E., pp. 44-60.

Debarbieux, B. (2004). "De l'objet spatial à l'effet géographique", in Debarbieux B., Fourny M.-C. (eds.), L'effet Géographique. Construction Sociale, Appréhension Cognitive et Configuration Matérielle des Objets Géographiques, CNRS.

Deeb, R., Ooms, K. and De Maeyer, P. (2012). Typography in the Eyes of Bertin, Gender and Expertise Variation. The Cartographic Journal. 49. pp. 176-185.

Deeb, R., Ooms, K., Vanopbroeke, V. and De Maeyer, P. (2014). Evaluating the Efficiency of Typographic Design: Gender and Expertise Variation. The Cartographic Journal. 51. pp. 75-86.

Dumont, M., Touya, G., Duchêne, C. (2016). A Comparative Study of Existing Multi-Scale Maps: What Content at Which Scale?, Proceedings of the 9th International Conference on Geographic Information Science (GIScience 2016), pp. 92-95.

Fairbairn, D. J. (1993). On the nature of cartographic text. The Cartographic Journal. 40. pp. 104-111.

Gerber, R. (1981). Competence And Performance In Cartographic Language. The Cartographic Journal. 18. pp. 104-111.

Guidero, E. (2013). Typography in the US Topo Redesign. Proceedings of the 26th International Cartographic Conference. 
Kent, A. J. and Vujakovic, P. (2009). Stylistic Diversity in European State 1:50 000 Topographic Maps. The Cartographic Journal. 46. pp. 179-213.

Lacoste, Y. (1980). Les Objets Géographiques. Cartes et figures de la terre. pp. 16-23. Centre Georges Pompidou, Paris.

Mathian, H. and Sanders, L. (2014). Spatio-temporal Approaches: Geographic Objects and Change Process. Spatio-temporal Approaches: Geographic Objects and Change Process.

Matlin, M. W. (2002). Cognitive Psychology and College-Level Pedagogy: Two Siblings That Rarely Communicate. New directions for teaching and learning, 2002 (89), p. 87-103.

Ory, J., Christophe, S., Fabrikant, S. and Bucher, B. (2015). How Do Map Readers Recognize a Topographic Mapping Style?. The Cartographic Journal. 52. pp. 193203.

Parsons E. (2014) Ambient Location: the where of everything. ePSI Platform Workshop: Make the Most of Geo Information, INSPIRE Conference. Aalborg University, Danemark.

Phillips, R. J. and Noyes, L. (1977). Searching for names in two city street map. Applied Ergonomics. 8. p.73-77.

Rylov, M. A. and Reimer, A. W. (2014). A comprehensive multi-criteria model for high cartographic quality Point-Feature label placement. Cartographica, 49. pp. 52-68.

Shimron, J. (1975). On Learning Maps, Office of Naval Research, Washington, D.C., 24.

Soltis, J. F. (1966). Seeing, Knowing and Believing, Allen \& Unwin, London.

Wilson, R., and Keil, F. (1999). The MIT Encyclopedia of the Cognitive Sciences. 